\title{
La prostaglandine D2
}

\section{Nouveaux rôles dans \\ la gonade embryonnaire et pathologique}

> La prostaglandine D2 (PGD2) fait partie de la famille des prostaglandines, molécules ubiquitaires de signalisation, liant des récepteurs transmembranaires couplés aux protéines $G$ et induisant différentes voies de transduction. Les prostaglandines PGE2 et PGF2 $\alpha$ ont un rôle reconnu dans l'ovulation, la fécondation et l'implantation; cependant, le (ou les) rôle(s) de la PGD2 dans les organes reproducteurs mâle ou femelle ne sont pas définis bien que la prostaglandine D synthase (PGDS), l'enzyme de synthèse de la PGD2, y soit détectée. Dans cet article, nous résumons les travaux récents mettant en évidence deux fonctions de la PGD2, dans la mise en place du testicule embryonnaire et dans l'inhibition de croissance des cellules ovariennes tumorales, via l'activation du facteur SOX9 de différenciation des cellules de Sertoli. <

\section{Les prostaglandines : synthèse et transduction du signal}

Les prostaglandines $(P G)$ sont de petites molécules dérivées d'acides gras poly-insaturés et appartiennent à la superfamille des eicosanoïdes. Le précurseur des prostanoïdes, l'acide arachidonique, est produit par l'hydrolyse des glycérophospholipides membranaires par la phospholipase A2 et, à un moindre degré, par la phospholipase C. L'acide arachidonique subit une oxydation puis une réduction par les enzymes COXI et COX2 (cyclooxygénases 1 et 2 également dénommées PGHSI et PGHS2, pour prostaglandin endoperoxydase $H$ synthases 1 and 2), pour être transformé en PGG2 (prostaglandin endoperoxydase G2) puis en PGH2 (prostaglandin endoperoxydase H2). La PGH2 est ensuite convertie par des prostaglandines synthases (PGDS, PGES, PGFS ou PGIS, ou TXAS) en prostaglandines, PGD2, PGE2, PGF2 $\alpha$, ou prostacyline ( $P G I 2)$ ou thromboxane A2 (TXA2) respectivement (Figure 1). Ces molécules sont sécrétées et activent des récepteurs à sept domaines transmembranaires, couplés aux protéines G, les GPCR (G protein coupled-receptor) (Figure 1). II en existe neuf membres, DP1 et DP2 (ou

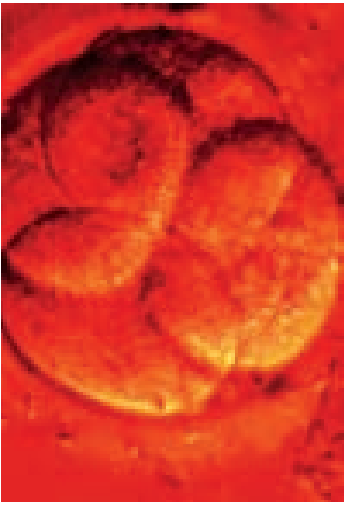

CRTH2) pour la PGD2, EP1-4 pour la PGE2, FP pour la PGF2 $\alpha$, IP pour la PGI2 et TP pour le TxA2, qui transduisent Développement et Pathologie de la Gonade, Département Génétique et Développement, Institut de Génétique Humaine (IGH), CNRS UPRI142, 141 , rue de la Cardonille, 34396 Montpellier Cedex 5 , France.

\section{boizet@igh.cnrs.fr}

S. Malki (adresse actuelle) :

Carnegie Institution,

Department of Embryology, Baltimore MD 21218, États-Unis. malki@ciwemb.edu des signaux différents via la production des seconds messagers AMPc ou IP3/ diacylglycérol/ $/ \mathrm{Ca}^{++}[1,2]$ (Figure 1).

Les prostaglandines sont impliquées dans de nombreux systèmes physiologiques, le système nerveux central, cardiovasculaire, gastro-intestinal, génito-urinaire, endocrine, respiratoire et immunitaire [2]. Ces molécules agissent localement de façon autocrine et/ou paracrine, et sont considérées comme des médiateurs proinflammatoires ou anti-inflammatoires selon la nature de la molécule et du récepteur activé, et le contexte cellulaire. Cette action est très complexe car les prostaglandines peuvent avoir des effets synergiques ou antagonistes pour un même processus physiologique, étant donnée la grande similitude de structure de ces molécules et aussi de leurs récepteurs.

D'un point de vue pharmacologique, elles constituent d'excellentes cibles thérapeutiques, puisque la synthèse des prostaglandines est impliquée dans de nombreuses maladies dont certains cancers, l'inflammation, des maladies cardiovasculaires et l'hypertension [2]. Les anti-inflammatoires non stéroïdiens, les NSAID (non steroidal anti-inflammatory drugs), qui inhibent l'action 
des enzymes COX1 et COX2 bloquant ainsi la synthèse des prostaglandines, sont déjà utilisés pour leurs activités anti-pyrétique, analgésique et anti-inflammatoire. Toutefois des effets secondaires de ces traitements ont été associés à l'inhibition de la synthèse des prostaglandines, puisque par exemple la PGE2 produite via COX1 ou COX2 a un rôle protecteur de l'intégrité de la muqueuse gastrique et des fonctions normales du rein, respectivement.

\section{La prostaglandine D2 (PGD2)}

\section{La synthèse de la prostaglandine D2}

La synthèse de la prostaglandine D2 est sous le contrôle spécifique de l'enzyme prostaglandine D synthase (PGDS). II en existe deux types, la Lipocaline-PGDS (L-PGDS), initialement identifiée dans le cerveau

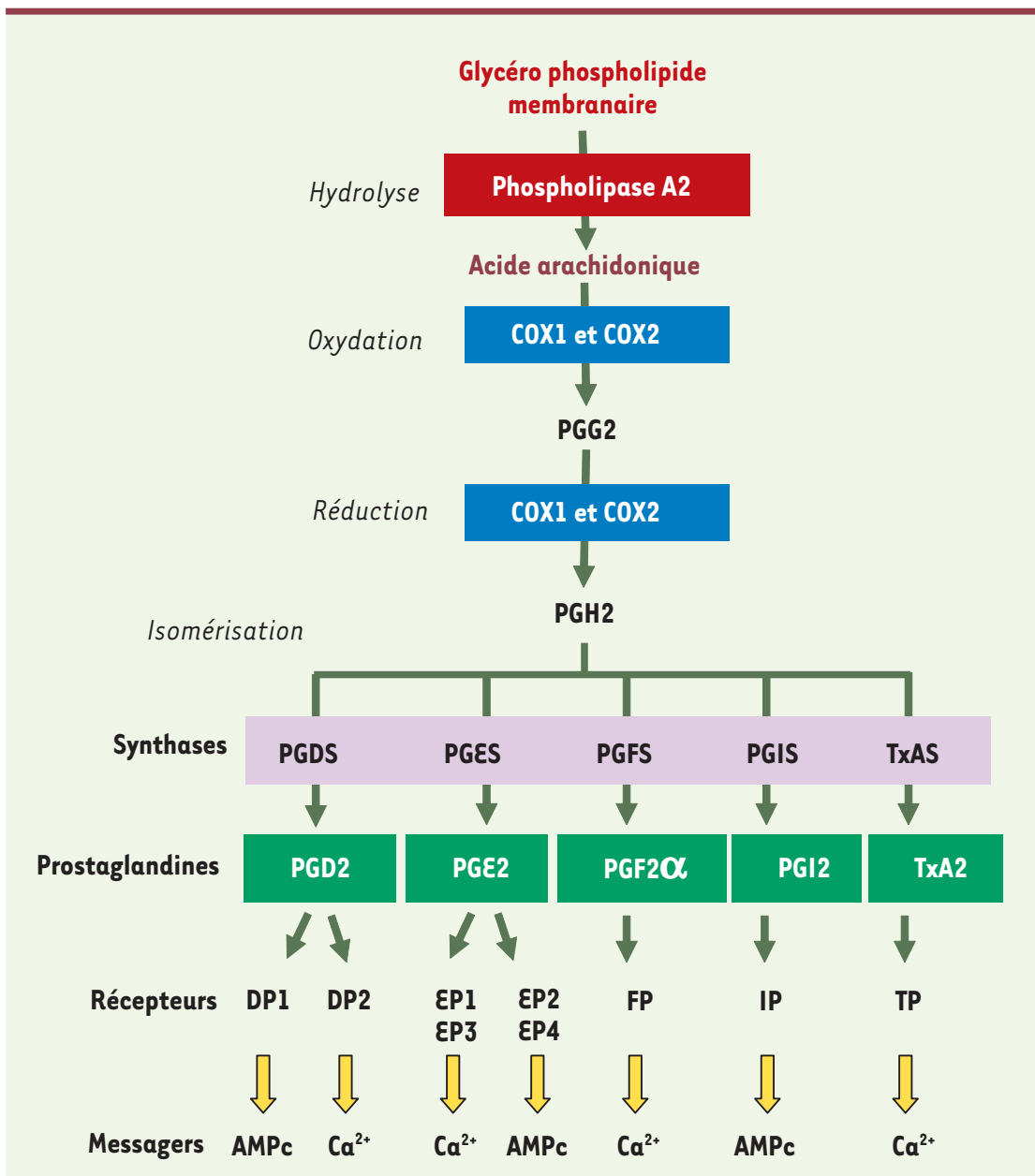

Figure 1. Synthèse et actions des prostaglandines. L'acide arachidonique est métabolisé par les enzymes COX1 et COX2 en PGG2 puis en PGH2 au cours d'une réaction en deux étapes (oxydation et réduction). La prostaglandine $\mathrm{PGH} 2$, relativement instable, est convertie enzymatiquement par des synthases spécifiques: thromboxane (TxAS), PGE, PGF, PGD et PGI synthases, en I'un des cinq prostanoïdes: TxA2, PGE2, PGF2 $\alpha, P G D 2$ et PGI2, respectivement. Chaque prostanoïde interagit avec des membres distincts de la famille des récepteurs membranaires couplés aux protéines $G$. Ces récepteurs (IP, DP1, DP2, EP l-4, FP, IP et TP) activent ensuite différentes réponses et voies de signalisation. de rat, dont la fonction est indépendante du tripeptide glutathion, et la PGDS hématopoïétique (H-PGDS), initialement identifiée dans la rate de rat, dont la fonction est dépendante de ce tripeptide. La PGD2 a une structure instable et subit rapidement une déshydratation pour former une grande variété de métabolites: 15-désoxy-PGD2, PGJ2 ou 15-désoxy-PGJ2.

La L-PGDS est le premier membre de la superfamille des lipocalines, protéines sécrétées dans les espaces extracellulaires, qui lient et transportent les petites molécules lipophiles (rétinol, $\beta$-lactoglobuline, pigments biliaires ou hormones thyroïdiennes), à exercer une activité enzymatique [3]. La L-PGDS a donc une double fonction d'enzyme d'isomérisation de la PGH2 en PGD2 et de transporteur. Cette enzyme est produite dans le système nerveux central (cerveau, moelle épinière, ganglions rachidiens dorsaux), dans les organes génitaux mâles (testicule, épididyme, prostate) et dans le cœur. Associée au réticulum endoplasmique et à la membrane nucléaire externe, elle jouera son rôle d'enzyme productrice de PGD2. De ces tissus, elle sera sécrétée dans de nombreux fluides, le fluide cérébrospinal (où elle a été confondue avec la protéine $\beta$-traceur), le plasma séminal, l'ascite, le sérum, l'urine, le liquide amniotique, où elle agira comme transporteur de molécules lipophiles [3, 4].

La H-PGDS est un membre de la classe des enzymes glutathion-S-transférases, enzymes cytosoliques jouant un rôle dans la détoxification enzymatique. Malgré la forte homologie de sa séquence primaire dans les différentes espèces, le profil d'expression tissulaire est très divergent: tissus périphériques, rate, thymus, moelle osseuse, tube digestif, oviducte chez le rat [5], peau et oviducte chez la souris [5] et placenta, poumons, foie fœtal, cœur, cerveau, mastocystes, lymphocytes de type Th2 et cellules présentatrices d'antigènes chez l'homme $[5,6]$. La H-PGDS est l'enzyme-clé de la synthèse de la PGD2 dans le système immunitaire et les mastocytes [7].

\section{Régulation de la synthèse \\ de la prostaglandine D2}

La synthèse de la PGD2, comme celle de l'ensemble des prostaglandines, est régulée via le couplage fonctionnel et différen- 
tiel des enzymes COX1 et COX2 aux différentes prostaglandines synthases, spécifiquement l'enzyme PGDS pour la PGD2 [8]. Le rôle biologique de chaque isoforme COX1 ou COX2 a été défini grâce aux modèles de souris invalidées pour chacun de ces gènes ou pour les deux gènes $[9,10]$. COXl, comme COX2, est impliquée dans le développement embryonnaire (fermeture du ductus arteriosus) et dans certaines pathologies, inflammation, développement de tumeurs intestinales et ulcères gastriques, mais COX2 est l'isoforme principale impliquée dans le développement post-natal du rein et dans le système de reproduction femelle (voir plus loin).

L'expression de COXl est relativement stable dans tous les tissus et cellules. Ce n'est pas le cas de celle de COX2, qui est induite, entraînant la production de prostaglandine PGE2 essentiellement, par de nombreux facteurs de croissance, des cytokines pro-inflammatoires (IL-1 [interleukine-1]), TNF $\alpha$ [tumor necrosis factor- $\alpha$ ]). L'expression du gène COX2 est par ailleurs inhibée par les glucocorticoïdes et les cytokines anti-inflammatoires comme I'IL-4 et I'IL-10, via la production de l'inhibiteur $\mathrm{I}-\mathrm{kB}$ et via la modification de la stabilité de I'ARN messager COX2 [9, 10].

L'expression de la L-PGDS est également sous le contrôle de nombreuses voies de signalisation, la protéine kinase C (PKC) [11], les œstrogènes [12], I'IL-1 $\beta$, RasGRP4 (ras guanine nucleotide-releasing protein 4) [13], ces régulations étant très spécifiques d'un type cellulaire à l'autre. Une étude in vitro montre la régulation de la L-PGDS par la progestérone et les métabolites de la vitamine A et l'hormone thyroïdienne T3 dans les cellules de Sertoli adultes [14]. La synthèse de la PGD2 est donc régulée via une multitude de voies de régulation spécifiques du contexte cellulaire.

\section{La transduction du signal de la prostaglandine D2}

La PGD2 se lie à deux récepteurs GPCR, le récepteur DP1 [15] et le récepteur DP2 ou CRTH2 (chemoattractant receptor-homologous molecule expressed on Th2 cells) découvert plus récemment [16] (Figure 1). L'activation du récepteur DPl couplé à la protéine Gs induit la production du second messager AMPc stimulant la voie de la protéine kinase A (PKA) et induit également un flux calcique entrant [15]. L'activation du récepteur CRTH2 ou DP2, couplé à la protéine Gi, inhibe la production d'AMPc [16] et induit une mobilisation intracellulaire de $\mathrm{Ca}^{2+}$ due à la production d'inositol triphosphate [4]. Selon le type cellulaire, les effets de la transduction du signal PGD2 via l'activation de ces deux récepteurs peuvent être synergiques ou antagonistes [2].

\section{Les rôles de la prostaglandine D2}

La PGD2 est activement produite dans de nombreux organes mais c'est le prostanoïde le plus abondant dans le système nerveux central et les voies respiratoires des mammifères [3]. Des rôles essentiels dans la perception de la douleur, le sommeil, l'asthme allergique, l'inflammation et la reproduction (cette fonction sera développée dans le paragraphe suivant), ont été définis grâce à l'analyse des souris transgéniques $L-P G D S$ ou des souris invalidées pour le gène $L-P G D S$ et pour les gènes codant les récepteurs DPl et CRTH2.

- La PGD2 produite par la L-PGDS est essentielle pour la perception de la douleur tactile induite par la PGE2 [17] ; l'équilibre entre PGE2 et
PGD2 représentant un élément pivot pour l'induction et la suppression de la douleur.

- Le sommeil induit par la PGD2 est similaire au sommeil physiologique [18] ; la PGD2 régule le sommeil via l'activation de son récepteur DPl et via une augmentation de la concentration d'adénosine extracellulaire agissant sur son récepteur $A_{2 A} R[17,19]$. Au contraire, la PGE2 active le système histaminergique via le récepteur HIR pour induire l'éveil.

- Après une stimulation allergique, la production d'IgE active les mastocytes qui sécrétent environ 50 médiateurs chimiques différents dont la PGD2, qui vont tous contribuer à la réponse allergique [20]. La PGD2 induit la production d'histamine puis une vasodilatation dans la muqueuse nasale, une bronchoconstriction et le recrutement et l'activation des lymphocytes Th2 et des éosinophiles via les récepteurs DP1, TP et CRTH2 respectivement $[21,22]$. Les deux récepteurs DPl et CRTH2 ont donc des rôles complémentaires dans la médiation de la réponse allergique [23]. La production de PGD2 par les mastocytes est un processus dépendant des deux isoformes COX1 et COX2, et pourtant les anti-inflammatoires non stéroïdiens sont inefficaces dans le traitement de l'asthme allergique. Ces médicaments pourraient en fait inhiber la production de PGE2 qui antagonise l'effet de la PGD2 par ses propriétés bronchodilatatrices [22]. Ces différentes données obtenues chez l'animal ont été validées par l'identification de variants génétiques de DPl et CRTH2 chez des patients ayant une prédisposition allergique [22].

- Les prostaglandines (PG\&2, PGI2, PGD2), en synergie avec d'autres médiateurs comme l'histamine, sont impliquées dans le processus de l'inflammation. Des effets opposés sur différents types cellulaires du système immunitaire, cellules dendritiques, lymphocytes Th2, polynucléaires basophiles et éosinophiles, ont été décrits et attribués à l'activation anti-inflammatoire de DPl (augmentation du taux d'AMPc) et pro-inflammatoire de CRTH2 (mobilisation du $\mathrm{Ca}^{2+}$ intracellulaire). L'effet anti-inflammatoire de la PGD2 a été attribué en grande partie à la 15d-PGJ2, un métabolite de la PGD2, qui est un ligand du récepteur nucléaire PPAR (peroxisome proliferator-activated receptor $\gamma$ ). La PGD2, via 15d-PGJ2 et $\operatorname{PPAR} \gamma$, est capable de supprimer la réponse immunogène en inhibant la production des cytokines (TNF $\alpha, \mathrm{IL}-1 \alpha$, IL-6) ou des médiateurs (iNOS) pro-inflammatoires [4]. De plus, la résolution de l'inflammation s'accompagne d'un shift de la biosynthèse de la prostaglandine $\varepsilon$ synthase (PGES) vers celle de la prostaglandine $D$ synthase (PGDS) [24]; la production résultante de PGD2 et de son métabolite $15 \mathrm{~d}-\mathrm{PGJ} 2$ contribuant à la résolution de l'inflammation. 
La PGD2 et ses métabolites sont donc des médiateurs-clé des maladies inflammatoires dans lesquelles un double rôle de promoteur et d'inhibiteur du processus inflammatoire est mis en évidence, selon l'espèce animale, l'organe, le stimulus inflammatoire et le récepteur activé $[2,4]$.

\section{La prostaglandine D2 dans le système de reproduction}

\section{Rôles dans les gonades adultes}

Dans les organes reproducteurs femelles, les prostaglandines jouent un rôle à de nombreuses étapes de la reproduction. Les souris défi-

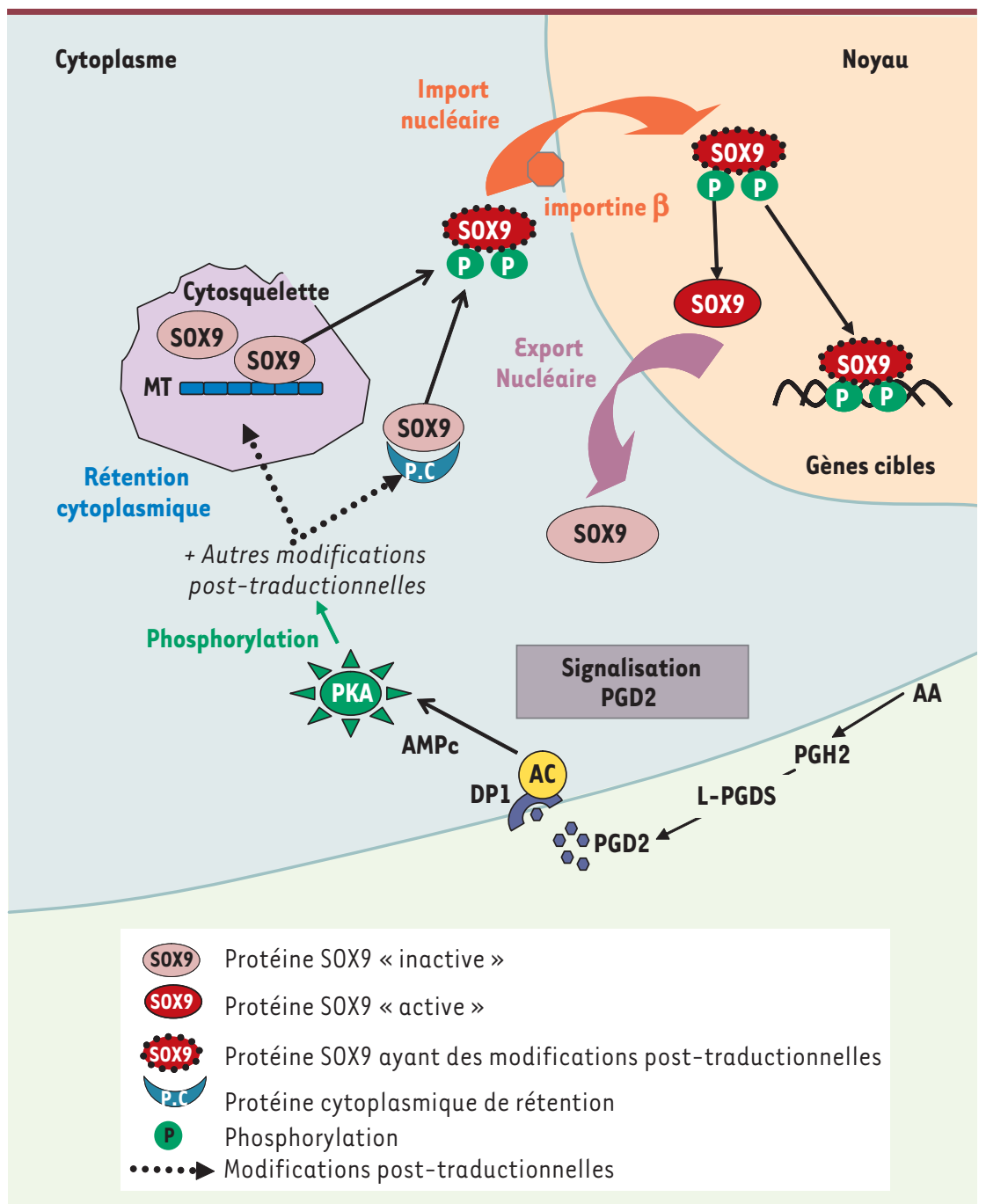

Figure 2. Activation de la translocation nucléaire du facteur SOX9 de différenciation testiculaire par la PGD2. Dans la gonade embryonnaire indifférenciée mâle ou femelle, la protéine SOX9 est localisée dans le cytoplasme des cellules somatiques: I'interaction de SOX9 avec le réseau de microtubules contribue à sa rétention cytoplasmique. La PGD2 synthétisée se lie à son récepteur DP1 couplé à l'adénylcyclase, activant la protéine kinase A (PKA). La phosphorylation de SOX9 sur deux sérines (Ser6l et Ser181) par la PKA interrompt l'interaction de SOX9 avec la tubuline et favorise son interaction avec la protéine de transport nucléaire importine $\beta$. L'import nucléaire de SOX9 lui permet de se lier aux promoteurs de ses gènes cibles permettant l'induction de la différenciation des cellules de Sertoli. cientes pour le gène COX2 ont des défauts dans les processus d'ovulation, de fertilisation et d'implantation [10]. L'invalidation du récepteur prostanoïde EP2 induit les mêmes phénotypes, démontrant l'implication de la PGE2 dans ces processus. COX2, PGE2 et EP2, synthétisées dans les cellules folliculaires du cumulus en réponse aux gonadotropines, induisent la maturation du follicule et de l'ovocyte nécessaire à la fertilisation et à l'ovulation [25]. Le défaut d'implantation embryonnaire dans les souris $\mathrm{COX2}^{-/-}$est annulé par la PGJ2 et par des agonistes du récepteur PPAR $\gamma$, indiquant que PGJ2, via PPAR $\gamma$, participe à ce processus. Quant à l'invalidation du gène COXI chez la souris, elle démontre l'importance de l'expression maternelle de COXl pour l'initiation du travail. C'est l'action de la PGF2 $\alpha$, fortement exprimée dans l'utérus, qui serait impliquée, via le récepteur FP, dans ce processus [25]. Des études in vitro ont montré que la PGF2 $\alpha$, exprimée par le corps jaune de l'ovaire de souris, est impliquée dans l'apoptose de ces cellules en l'absence de gestation [26]. La quantification du taux des prostaglandines dans le fluide folliculaire a montré que la production de PGE2 et PGF2 $\alpha$ augmente au cours de la phase folliculaire pré-ovulatoire; au cours de la phase lutéale, la production de la PGF2 $\alpha$ diminue tandis que celle de la PGE2 persiste et que celle de la PGD2 augmente [27].

Peu d'études analysent l'implication de la PGD2 dans la reproduction femelle. La H-PGDS et les deux récepteurs DPl et CRTH2 sont exprimés dans le placenta et la L-PGDS dans le liquide amniotique, indiquant un rôle dans la régulation de la communication fœto-placentaire [27]. Enfin, la PGD2 peut subir une déhydratation la transformant en précurseur de la PGJ2 qui active la voie du PPAR $\gamma$ impliquée dans la stéroïdogenèse dans les cellules de la thèque.

Chez le mâle, aucun modèle de souris établi à ce jour ne rend compte d'un défaut de la reproduction mâle. Une étude in vitro récente a identifié l'expression des deux PGDS et du récepteur DPI dans le testicule humain: la H-PGDS produite par les mastocytes du testicule et la L-PGDS produite par les cellules de 
Leydig et DPl exprimé également par ces cellules [28]. Par ailleurs, la PGD2 induit une augmentation de la production de testostérone par des cellules de Leydig isolées de testicule de hamster [28], suggérant un rôle pour la L-PGDS en tant qu'enzyme de production de la PGD2 dans les cellules de Leydig. Dans l'épididyme, COX2 induit l'expression de PGDS qui, en produisant la PGD2, protège les cellules de l'apoptose [29]. La présence de l'enzyme L-PGDS dans le liquide séminal, qui reflète l'expression du gène dans la queue de l'épididyme et de la prostate, souligne le rôle de la L-PGDS dans la spermatogenèse. Dans ce processus, le rôle de la L-PGDS serait celui d'un transporteur de molécules lipophiles, dont l'acide rétinoïque [30].

\section{La prostaglandine D2 est un facteur autocrine} dans la formation de la gonade embryonnaire mâle

Au cours du développement testiculaire, à $11,5 \mathrm{dpc}$ (days post-coïtum) chez la souris, la L-PGDS est spécifiquement exprimée dans les gonades mâles [31, 32], dans les cellules germinales et les cellules de Sertoli [14, 33, 34], expression postérieure à celle de Sry et Sox9, les deux facteurs-clés de la détermination testiculaire $[35,36]$. Sur le plan moléculaire, avant le pic d'expression du gène Sry à $11,5 \mathrm{dpc}$, le transcrit Sox 9 est très faiblement exprimé dans la gonade mâle ou femelle [37]. La protéine Sox9 est alors localisée dans le cytoplasme des cellules pré-Sertoli des deux sexes $[37,38]$. Lorsque le gène Sry atteint son pic d'expression à $11,5 \mathrm{dpc}$, le transcrit Sox9 augmente chez le mâle et de façon concomitante, la protéine Sox9 est transportée dans le noyau. Nous avons mis en évidence un rôle de la PGD2 dans l'induction de la translocation nucléaire de Sox 9 dans les celIules de Sertoli [33] et dans l'activation de son expression (données non publiées et [31]). Par différentes études pharmacologiques, nous avons montré que l'accumulation nucléaire de Sox9 est médiée via le récepteur DPI (Figure 2). La translocation nucléaire de Sox9 est induite après sa phosphorylation par la protéine kinase dépendante de I'AMPc (PKA) sur deux résidus sérine; cette phosphorylation augmente l'interaction de Sox 9 avec la protéine de transport nucléocytoplasmique, l'importine $\beta$ et interrompt l'interaction de Sox9 avec le réseau de microtubules, favorisant ainsi sa translocation nucléaire [39]. Ces résultats identifient donc la PGD2 via son récepteur DPl comme facteur autocrine de l'activation de Sox9 et par conséquent, de la différenciation des cellules de Sertoli. L'analyse des gonades des souris invalidées pour le gène L-PGDS indique un défaut de la mise en place des tubules séminifères, confirmant les expériences in vitro précédemment décrites (résultats non publiés). L'activation de cette voie de signalisation est concomitante à la mise en place de la boucle d'autorégulation entre Fgf9 et Sox 9 contribuant à la surexpression de Sox 9 dans la cellule de Sertoli [40].

Figure 3. Expression de SOX9 dans les tumeurs ovariennes et rôle de la voie PGDS/SOX9 dans les cellules ovariennes tumorales. A. SOX9 est détectée par immunohistochimie avec un anticorps spécifique anti-S0X9 dans deux biopsies ovariennes humaines de tumeurs épithéliale (adénocarcinome séreux) et de la granulosa (tumeur granulosa) (flèche: SOX9 nucléaire, tête de flèche: SOX9 cytoplasmique). B. La stimulation de cellules ovariennes tumorales en culture induit, via le récepteur DP1, l'expression de SOX9 (réponse précoce : 30 minutes à 1 heure) induisant plus tardivement l'apoptose des cellules (24 heures) et inhibant leur croissance (48 à 72 heures).

\section{La prostaglandine D2}

a un rôle anti-prolifératif et pro-apoptotique dans l'ovaire pathologique

Les mécanismes moléculaires et cellulaires impliqués dans l'étiologie du cancer de 
l'ovaire restent mal connus mais l'implication des oestrogènes dans la progression des tumeurs ovariennes semble bien établie [41]. Par ailleurs, un rôle important des prostaglandines endoperoxydases COX1 et COX2, de la prostaglandine $\varepsilon 2$ (PGE2) et de son enzyme de synthèse la prostaglandine $\varepsilon$ synthase (PGES), a été démontré dans la tumorigenèse ovarienne [42-44].

Nos études récentes ont montré une forte expression des partenaires de la voie de signalisation embryonnaire mâle L-PGDS/SOX9 dans des biopsies de tumeurs ovariennes humaines de différents types histologiques [45] (Figure 3A). Le traitement par la PGD2 de deux lignées ovariennes tumorales d'origine épithéliale (BGl) ou provenant de la granulosa (Cov434) induit une diminution de leur croissance et une augmentation de leur apoptose, via le récepteur DPl et l'activation de l'expression de SOX9 (Figure 3B) [45]. Une telle inhibition des cellules ovariennes KF (dérivées d'un adénocarcinome séreux humain) a été rapportée antérieurement in vitro [46] et in vivo dans des expériences de xénogreffes [47]. Par ailleurs, l'activation de l'expression de la L-PGDS par l'acide rétinoïque est responsable de l'inhibition de croissance des cellules ovariennes tumorales $3 \mathrm{AO}$ en culture [48]. À l'inverse des autres voies relayées par les prostaglandines, la voie PGDS/PGD $/$ SOX9 a donc un rôle de suppresseur des tumeurs ovariennes et pourrait être en équilibre avec la voie PGES/PGE2 qui, elle, est tumorigène.

\section{Conclusions}

Ces travaux récents sur la mise en place de la gonade embryonnnaire et sur l'ovaire pathologique ont mis en évidence de nouveaux rôles pour la PGD2 et une relation fonctionnelle du gène $P G D S$ codant l'enzyme prostaglandine $D$ synthase (PGDS) avec le facteur de transcription Sox9, facteur de différenciation des cellules de Sertoli. Nous avons identifié la L-PGDS comme une nouvelle pièce dans le puzzle de la détermination sexuelle mâle, la PGD2 constituant un facteur autocrine de l'activation de Sox9. Des études supplémentaires sont nécessaires pour déterminer si cette voie de signalisation PGDS/Sox9 fait partie de la boucle de régulation Fgf9/Sox9 mise en évidence récemment [40] ou si elle représente une voie parallèle. Nous avons mis en évidence une expression de cette voie de signalisation PGDS/Sox9 dans les cancers de l'ovaire, et sa stimulation induit une diminution de prolifération et une augmentation de l'apoptose des cellules ovariennes tumorales. Cette étude a permis la dissection moléculaire des tumeurs ovariennes dont la fréquence est en constante progression et la mise en évidence du rôle des facteurs de différenciation testiculaire, prostaglandine D synthase et Sox9, comme nouveaux marqueurs diagnostiques ou pronostiques dans le cancer de l'ovaire. Elle permet aussi d'envisager l'utilisation d'activateurs spécifiques de cette voie de signalisation pour retarder l'évolution de la pathologie et ainsi d'élaborer de nouvelles stratégies thérapeutiques. $\diamond$

\section{SUMMARY}

Prostaglandin D2:

new roles in the embryonic and pathological gonad

Prostaglandin D2 (PGD2) belongs to the superfamily of ubiquitous signalling molecules, the prostaglandins; these bind to specific G-coupled transmembrane receptors, inducing various transduction pathways. Prostaglandins PGE2 and PGF2 $\alpha$ have several identified functions during ovulation, fecondation and embryo implantation. However, the roles of PGD2 within the male or female reproductive organs are still largely unknown, even though the PGD2-producing enzyme, prostaglandin D synthase (PGDS), is detected in these organs. In this study, we summarize recent data highlighting new functions of PGD2 in the onset of testicular embryogenesis and in the growth inhibition of ovarian cancer cells. In both cases, PGD2 acts by activating the function of the Sertoli cell differentiating factor S0X9. $\diamond$

\section{RÉFÉRENCES}

1. Breyer RM, Bagdassarian CK, Myers SA, Breyer MD. Prostanoid receptors : subtypes and signaling. Annu Rev Pharmacol Toxicol 2001 ; 41 : 661-90.

2. Hata AN, Breyer RM. Pharmacology and signaling of prostaglandin receptors: multiple roles in inflammation and immune modulation. Pharmacol Ther 2004 ; 103 : 147-66.

3. Urade Y, Hayaishi 0 . Biochemical, structural, genetic, physiological, and pathophysiological features of lipocalin-type prostaglandin D synthase. Biochim Biophys Acta 2000 ; 1482 : 259-71.

4. Herlong JL, Scott TR. Positioning prostanoids of the $D$ and J series in the immunopathogenic scheme. Immunol Lett 2006 ; 102 : 121-31.

5. Kanaoka Y, Fujimori K, Kikuno R, et al. Structure and chromosomal localization of human and mouse genes for hematopoietic prostaglandin D synthase. Conservation of the ancestral genomic structure of sigma-class glutathione S-transferase. Eur J Biochem $2000 ; 267: 3315-22$.

6. Tanaka K, Ogawa K, Sugamura K, et al. Cutting edge : differential production of prostaglandin D2 by human helper T cell subsets. J Immunol 2000 ; $164: 2277-80$.

7. Urade $Y$, Hayaishi 0 . Prostaglandin $D$ synthase : structure and function. Vitam Horm $2000 ; 58: 89-120$.

8. Ueno N, Takegoshi Y, Kamei D, et al. Coupling between cyclooxygenases and terminal prostanoid synthases. Biochem Biophys Res Commun 2005; 338: 70-6.

9. Morita I. Distinct functions of COX-1 and COX-2. Prostaglandins Other Lipid Mediat $2002 ; 68-69: 165-75$.

10. Loftin CD, Tiano HF, Langenbach R. Phenotypes of the COX-deficient mice indicate physiological and pathophysiological roles for COX-1 and COX-2. Prostaglandins Other Lipid Mediat 2002 ; 68-69: 177-85.

11. Fujimori K, Kadoyama K, Urade Y. Protein kinase C activates human lipocalintype prostaglandin $D$ synthase gene expression through de-repression of notch-HES signaling and enhancement of AP-2 beta function in brain-derived TE671 cells. J Biol Chem $2005 ; 280$ : 18452-61.

12. Mong JA, Devidze N, Frail DE, et al. Estradiol differentially regulates lipocalintype prostaglandin $D$ synthase transcript levels in the rodent brain : evidence from high-density oligonucleotide arrays and in situ hybridization. Proc Natl Acad Sci USA $2003 ; 100: 318-23$

13. Li L, Yang Y, Stevens RL. RasGRP4 regulates the expression of prostaglandin D2 in human and rat mast cell lines. J Biol Chem 2003; $278: 4725-9$.

14. Samy ET, Li JC, Grima J, et al. Sertoli cell prostaglandin D2 synthetase is a multifunctional molecule : its expression and regulation. Endocrinology 2000 ; $141: 710-21$.

15. Boie Y, Sawyer N, Slipetz DM, et al. Molecular cloning and characterization of the human prostanoid DP receptor. J Biol Chem 1995; 270 : 18910-6.

16. Hirai H, Tanaka K, Yoshie 0 , et al. Prostaglandin D2 selectively induces chemotaxis in Thelper type 2 cells, eosinophils, and basophils via seventransmembrane receptor CRTH2.J Exp Med 2001 ; 193 : 255-61.

17. Kobayashi T, Narumiya S. Function of prostanoid receptors : studies on knockout mice. Prostaglandins Other Lipid Mediat $2002 ; 68-69$ : 557-73.

18. Pinzar $\varepsilon$, Kanaoka $Y$, Inui T, et al. Prostaglandin D synthase gene is involved in the regulation of non-rapid eye movement sleep. Proc Natl Acad Sci USA $2000 ; 97: 4903-7$.

19. Huang ZL, Urade $Y$, Hayaishi 0 . Prostaglandins and adenosine in the regulation of sleep and wakefulness. Curr Opin Pharmacol $2007 ; 7: 33-8$.

20. Rolin S, Masereel B, Dogne JM. Prostanoids as pharmacological targets in COPD and asthma. Eur J Pharmacol $2006 ; 533: 89-100$.

21. Matsuoka T, Hirata M, Tanaka H, et al. Prostaglandin D2 as a mediator of allergic asthma. Science $2000 ; 287: 2013-7$.

22. Pettipher R, Hansel TT, Armer R. Antagonism of the prostaglandin D2 receptors DP1 and CRTH2 as an approach to treat allergic diseases. Nat Rev Drug Discov $2007 ; 6: 313-25$. 
23. Kostenis $\varepsilon$, Ulven T. Emerging roles of DP and CRTH2 in allergic inflammation. Trends Mol Med $2006 ; 12$ : 148-58.

24. Schuligoi R, Grill M, Heinemann A, et al. Sequential induction of prostaglandin $\varepsilon$ and $D$ synthases in inflammation. Biochem Biophys Res Commun 2005; $335: 684-9$.

25. Kobayashi T, Narumiya S. Prostanoids in health and disease ; lessons from receptor-knockout mice. Adv Exp Med Biol 2002 ; 507 : 593-7.

26. Hasumoto K, Sugimoto $Y$, Yamasaki $A$, et al. Association of expression of mRNA encoding the PGF2 alpha receptor with luteal cell apoptosis in ovaries of pseudopregnant mice. J Reprod Fertil 1997 ; 109 : 45-51.

27. Lumsden MA, Kelly RW, Templeton AA, et al. Changes in the concentration of prostaglandins in preovulatory human follicles after administration of hCG.J Reprod Fertil 1986; 77 : 119-24.

28. Schell C, Frungieri MB, Albrecht M, et al. A Prostaglandin D2 system in the human testis. Fertil Steril $2007 ; 88: 233-6$.

29. Cheuk BL, Chew SB, Fiscus RR, Wong Py. Cyclooxygenase- 2 regulates apoptosis in rat epididymis through prostaglandin D2. Biol Reprod 2002; 66:374-80.

30. Saito S, Tsuda H, Michimata T. Prostaglandin D2 and reproduction. Am J Reprod Immunol 2002; $47: 295-302$.

31. Wilhelm D, Martinson F, Bradford S, et al. Sertoli cell differentiation is induced both cell-autonomously and through prostaglandin signaling during mammalian sex determination. Dev Biol 2005 ; 287 : 111-24.

32. Wilhelm D, Hiramatsu R, Mizusaki $H$, et al. SOX9 regulates prostaglandin D synthase gene transcription in vivo to ensure testis development. J Biol Chem $2007 ; 282$ : 10553-60.

33. Malki S, Nef S, Notarnicola C, et al. Prostaglandin D2 induces nuclear import of the sex-determining factor SOX9 via its CAMP-PKA phosphorylation. EMBO $2005 ; 24: 1798-809$

34. Nef S, Schaad 0 , Stallings NR, et al. Gene expression during sex determination reveals a robust female genetic program at the onset of ovarian development. Dev Biol 2005 ; 287 : 361-77.

35. Vidal VP, Chaboissier MC, de Rooij DG, Schedl A. Sox9 induces testis development in XX transgenic mice. Nat Genet $2001 ; 28: 216-7$
36. Barrionuevo F, Bagheri-Fam S, Klattig J, et al. Homozygous inactivation of Sox 9 causes complete XY sex reversal in mice. Biol Reprod $2006 ; 74: 195-201$.

37. Morais da Silva S, Hacker A, Harley V, et al. Sox9 expression during gonadal development implies a conserved role for the gene in testis differentiation in mammals and birds. Nat Genet 1996 ; $14: 62-8$.

38. Gasca S, Canizares J, De Santa Barbara P, et al. A nuclear export signal within the high mobility group domain regulates the nucleocytoplasmic translocation of SOX9 during sexual determination. Proc Natl Acad Sci USA 2002 ; 99 : 11199-204.

39. Malki S, Berta P, Poulat F, Boizet-Bonhoure B. Cytoplasmic retention of the sex-determining factor SOX9 via the microtubule network. Exp Cell Res $2005 ; 309:$ 468-75.

40. Kim Y, Kobayashi A, Sekido R, et al. Fgf9 and wnt4 act as antagonistic signals to regulate Mammalian sex determination. PLoS Biol $2006 ; 4$ : el 87.

41. Pujol P, Rey JM, Nirde P, et al. Differential expression of estrogen receptor-alpha and -beta messenger RNAs as a potential marker of ovarian carcinogenesis. Cancer Res 1998 ; $58: 5367-73$

42. Munkarah AR, Morris R, Baumann $P$, et al. Effects of prostaglandin $\varepsilon(2)$ on proliferation and apoptosis of epithelial ovarian cancer cells. J Soc Gynecol Investig 2002; 9 : 168-73.

43. Denkert C, Kobel M, Pest $S$, et al. Expression of cyclooxygenase 2 is an independent prognostic factor in human ovarian carcinoma. Am J Pathol 2002; 160:893-903.

44. Daikoku T, Wang D, Tranguch $S$, et al. Cyclooxygenase- 1 is a potential target for prevention and treatment of ovarian epithelial cancer. Cancer Res $2005 ; 65: 3735-44$

45. Malki S, Bibeau F, Notarnicola C, et al. Expression and biological role of the prostaglandin D synthase/SOX9 pathway in human ovarian cancer cells. Cancer Lett $2007 ; 255: 182-93$

46. Kikuchi $\mathrm{Y}$, Kita T, Miyauchi M, et al. Inhibition of human ovarian cancer cell proliferation in vitro by neuroendocrine hormones. Gynecol Oncol 1989; $32: 60-4$.

47. Kikuchi Y, Miyauchi M, Iwano I, et al. Adjuvant effects of prostaglandin D2 to cisplatin on human ovarian cancer cell growth in nude mice. Eur J Cancer Clin Oncol 1988; 24 : 1829-33.

48. Su B, Guan M, Xia J, Lu Y. Stimulation of lipocalin-type prostaglandin D synthase by retinoic acid coincides with inhibition of cell proliferation in human $3 \mathrm{AO}$ ovarian cancer cells. Cell Biol Int 2003 ; $27: 587-92$.

TIRÉS À PART

B. Boizet-Bonhoure
Cos

CRTER NATONAL. OELARFCENACHI SCENTHFOE.

\title{
CNRS Formation Entreprises
}

\author{
du 12 au 16 mai 2008 Separation des proteines par electrophorese bidimensionnelle \\ a PARIS (75)
}

du 16 au 18 jain 2009 Le risque chimique : connaissance et prévention niveau II a GIF SUR YVETTE (91)

du 16 au 20 juin 2008 Initiation theorique et pratique aux techniques de base de la biologie moleculaire A TOULOUSE (31)

\section{du 23 au 27 juin 2009 PCR quantitative en temps reel} AORSAY (92)

\section{du 17 au 19 septembre 2008 Analyse biointormatique des séquences nucléiques et proteiques} a PARIS (75)

du 23 au 26 septembve 2009 Uitramicrotomie : initiation theorique et pratique A ORSAY (97)

du 25 au 26 scptembre 2008 Le risque chimique : connaissance et prévention niveau 1 A GIF SUR YVETTE (97)

Centre de ressources en formation

Un protieme de lormation particulier 7 Nhevited pas a nous consuller

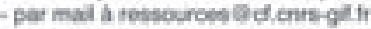
s par illehone au 01.6982 .4496 


\section{Ateliers de formation 2008}

Renseignements et inscriptions :

Ateliers de formation Inserm

101, rue de Tolbiac

75654 Paris Cedex 13

Tél. : 33 (0)1 44236204 - Fax : 33 (0)1 44236293

ateliers@tolbiac.inserm.fr

\section{Atelier de formation $n^{\circ} 185$}

\section{Protéines intrinsèquement désordonnées et pathologies associées : prédiction, caractérisation et fonction}

Organisateurs : Richard Haser (IBCP, Lyon), Sonia Longhi (AFMB, Marseille)

\section{Phase I • Le point sur...}

19-20 mai 2008 • Saint-Raphaël

Objectifs - Chez les eucaryotes une forte proportion du génome code pour des protéines intrinsèquement désordonnées (PID). Les PID sont des protéines fonctionnelles dépourvues de structure II et III stable en conditions physiologiques. Elles sont principalement impliquées dans la régulation et la signalisation cellulaire. Le désordre structural est aussi une caractéristique d'un certain nombre de protéines impliquées dans des pathologies neurodégénératives sévères (Alzheimer, Parkinson...). L'objectif de cet Atelier est de familiariser les participants avec les concepts de désordre structural et de repliement induit. II permettra de faire le point sur le rôle de ces protéines, les mécanismes moléculaires contrôlant leur repliement, et les répercussions fonctionnelles de ces phénomènes et leurs relations avec diverses pathologies.

Public - Chercheurs, ingénieurs, enseignants, doctorants et post-doctorants dans les domaines de la biologie et médecine moléculaires, la biophysique, la biologie moléculaire, la biologie structurale.

Les conférences seront données en anglais.

Nombre maximum de participants : 80 .

Programme - Une première partie sera consacrée aux critères définissant le désordre structural, à son rôle fonctionnel et à sa prédiction par des approches bioinformatiques. Ensuite, les approches expérimentales pour l'étude du désordre et du repliement induit seront largement discutées à travers des exemples de PID bien caractérisées. Enfin, la relation entre désordre et pathologies associées sera discutée, en soulignant les approches thérapeutiques principales pour le traitement des maladies neurodégénératives.

\section{Phase II • Maîtrise technique 23-25 juin $2008 \cdot$ Lyon}

Programme • La première partie du stage aura pour but l'apprentissage de différents logiciels de prédiction et l'interprétation des résultats. La deuxième partie sera consacrée à la caractérisation des PID par dichroïsme circulaire, spectroscopie de fluorescence, diffusion de la lumière, diffusion des rayons $X$ aux petits angles (SAXS) et Résonance Magnétique Nucléaire (RMN).

Sélection • 10 participants seront sélectionnés parmi les participants de la phase I.

Avec la participation de - Martin Blackledge (Grenoble, France), Keith Dunker (Indianapolis, USA), David Eliezer (New York, USA), Monika Fuxreiter (Budapest, Hongrie), Patrice Gouet (Lyon, France), Sonia Longhi (Marseille, France), Richard Kriwacki (Memphis, USA), Guy Lippens (Lille, France), Anne Poupon (Orsay, France), Véronique Receveur-Bréchot (Marseille, France), Peter Tompa (Budapest, Hungary), Vladimir Uversky (Indianapolis, USA), Peter Wright (San Diego, USA).

Date limite d'inscription : 14 mars 2008 